\title{
Probabilistic Neural Network with GLCM and Statistical Measurements for Increasing Accuracy of Iris Recognition System
}

\author{
Dhia Alzubaydi, PhD \\ Computer Science Dept. \\ University of Al Mustansiriyah / Iraq - Baghdad
}

\author{
Shyma Akram Alrubaie \\ Computer Science Dept. \\ University of Al Mustansiriyah / Iraq - Baghdad
}

\begin{abstract}
The main advantage of biometric system in security mode is either in verification process or identification process for the persons. Iris recognition is one of the fast, accurate, reliable and secure biometric techniques for human identification. It provides automatic authentication of an individual based on the characteristics and unique features in iris structure. Thus the most important step in biometric system is the method of extract feature from pattern, especially in using Artificial Neural Networks (ANN) in the matching (recognition) process. There will be a close relationship between the type of network used and the method of extracting features. In this paper, three method of features extraction is tested using three types of GLCM based on number of angles for each type (2Ang, 3-Ang, 4-Ang) as First Order Statistics (FOS) and 10 statistical measures as Second Order Statistics (SOS) for each type with three models of PNN, so as the model created is dependent on number of classes $(20,25,30)$ in each model. Experimental results proved that third method (4ang-GLCM) of feature extraction with higher trained classes (30) had given best Recognition Rate with accuracy $94.43 \%$. Thus, experimental results have been indicated to the efficiency of the proposed system in recognition accuracy in comparison with the previous methods.
\end{abstract}

\section{Keywords}

Iris recognition system (IRS), Histogram Equalization (HE), Region of interest (ROI), Artificial Neural Network (ANN), Probabilistic Neural Network (PNN), Gray Level Cooccurrence Matrix (GLCM), First Order Statistics (FOS), Second Order Statistics (SOS).

\section{INTRODUCTION}

Biometric system is basically pattern recognition system which executes by obtaining biometric data from persons, then extracting feature from the acquired data, and comparing this feature with the set of templates saved in database [1]. The search for the ideal personal identification method with high accuracy has been the aspiration of modern science (Biometric). In principle; when using biometric system further subjects of privacy and security must be addressed carefully. Thus, biometrics technology provided reliable, effective, stable, and unique feature in the identification of the individual by his or her physiological or behavioral characteristics, beside the high level of belief in execution, accuracy, adapt-ability, and cost of biometric technologies for different applications. Lately, the increased use of it in: banks; financial exchanges; offices and other places that require the authentication of the individuals, recently became a vital element of any successful person identification solutions $[2,3,4]$.
Today, most technologies generally use the human iris because iris based authentication systems afford more advantages than any other biometric technologies as it offers an excellent recognition performance [5]. This fact would be proven from set of attributes for each biometric, these attributes are considered the basic measurement of biometrics and on this basis we define the effectiveness of the used biometrics. A previous research conducted on various biometrics methods that showed there is a wide variation in the ratio of availability of these attributes in each biometric (characteristic) therefore developed for each attribute three levels of measurement: High, Medium and Low as shown in Figure (1). Thus any biometric method that offer the best levels of the attributes considered the best characteristic, for this reason iris biometric provided acceptable levels of attributes, therefore it considered the best characteristic after the DNA biometric [4].

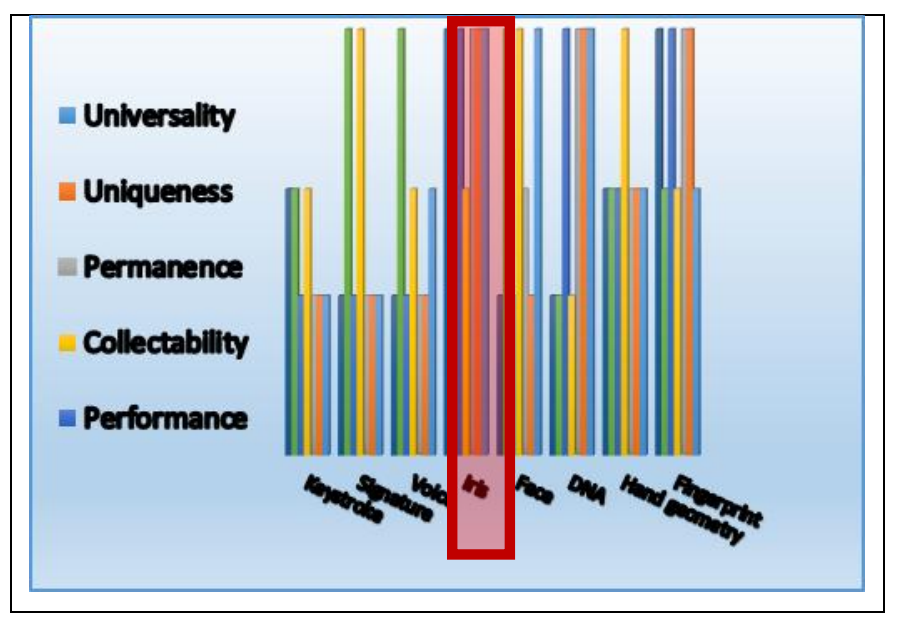

Fig 1: Basic Criteria for Biometrics System

In particularly, as a result of the evolution in the use of artificial neural networks in recognition system, the iris system is becoming more efficient [6]. IRS by use ANN; is similar to other biometric systems, it has three stages, registration stage, training stage and recognition (matching) stage [5]. There are four steps common between the Registration and Recognition stage, these as: preprocessing operations (localization, segmentation, normalization), and feature extraction. But Recognition stage contains additional step; matching (recognition) iris pattern step, the process of recognition utilizing Artificial Neural Network (ANN). While, Training stage is considered special stage of network working. So as, it is responsible for creating and simulating PNN with the data (features vectors) which previously stored in the database. In the registration process feature vector is extracted 
and stored in the database, while in the matching process, the extracted feature is compared with patterns training in net, Figure (2) shows block diagram of Iris Recognition.

The most challenges faced by the iris recognition is the selection of appropriate features and also in the selection of sufficient number of training samples of the network where there is an inverse relationship between the feature strength and the samples number. Thus fast, robust, and accurate implementation techniques are needed.

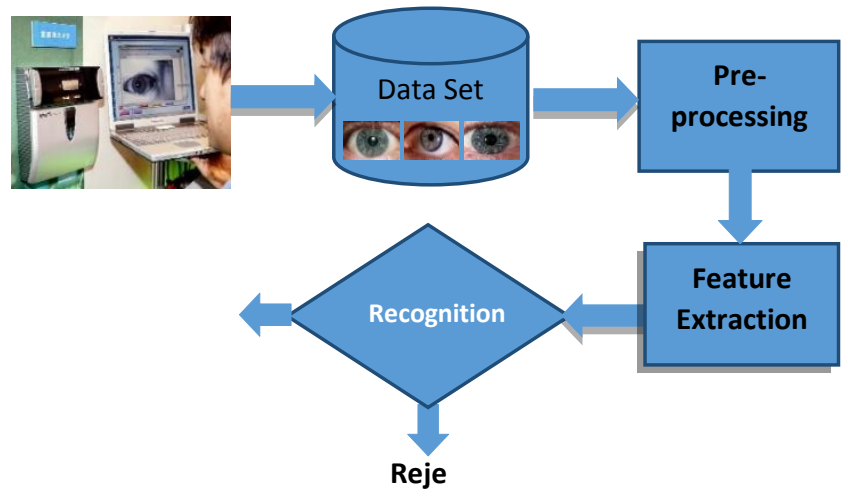

Fig 2: Block Diagram of Iris Recognition

This paper is organized as follows: section 2 presents preprocessing operations, section 3 presents feature extraction, section 4 creates an ANN, section 5 displays matching operation, section 6 illustrates the experimental results, section 7 displays conclusions, and finally references are shown in section 8 .

\section{PREPROCESSING OPERATION}

The proposed system is based on building a model of the iris recognition, which is used to extract distinguishing features from the ROI of iris, then these features are used in individuals recognition. Iris images of "CASIA-Iris-Interval" dataset is used in the proposed work which it's subset of "CASIA-Iris-Interval V4.0". In this set, images captured with close-up iris camera. The First Stage in IRS include: Preprocessing unit that contain three processes inside it, these processes are: localization of iris region which is distinguished by being less dark than pupil but it still darker when compared to sclera. The success of localization depends on the quality of eye image [4]. Localization algorithm performs faster than the traditional methods by converting the grayscale iris image to binary image using threshold value extracting from the maximum level of histogram image. Previously, enhancement by using (HE \& Unsharp Filter) will be made to increase clarity of image, then using morphological operations to remove noise and benefit from the chain code with Freeman computations [7] to calculate the center of mass that considered the pupil and iris center. After that the process of segmentation will be ready to split Collarette region of iris, region of Collarette consider the important part of the iris that is close to pupil and the clearest characteristics (feature) from all iris region [8]. The radius of iris Collarette has been estimated at 40 pixels that measured from the inner boundary of iris, collarette region separated from the image by using mask method to cover noise areas. Mask method [9] is suitable in the proposed system because necessary information is provided to do segment process, these information consist of the iris center, radius of pupil and radius of iris. These information will be used to create cover (mask) for pupil then make inverse mask for iris to determine region of interest ROI, while $50 \%$ of iris region has been able to localize the important region features of iris [6]. ROI would be extracted by isolating fixed rate from the upper region and less rate from the lower region. Lastly, normalization process to convert the image from Cartesian coordinates to Polar coordinates by using Daugman's Rubber Sheet Model. This model is used as basic model but with a small change to convert the image from donut shape to the rectangular shape, then get the important blocks from the iris area. The important block means: blocks extracted from the iris region that do not contain noise (eyelashes and eyelid) by exceeding the noise regions, the iris noise would previously covered with a black mask in the segmentation process, therefore it is facilitated to recognize this regions and exceed it. When the first step of normalization process is executed, the resulting image will been determined by the size $35 * 360$ pixels. In the proposed algorithm, three Blocks (First, Second, Third) are extracted from the iris region, which belong to: Upper Part of lower part of the iris region, Left Part, and Right Part. These blocks are shown in Figure (3) which contain clear features for extracting it in the next stage with size $18 * 84$ pixels for First Block, and 35*70 pixels for each of Second Block and Third Block.

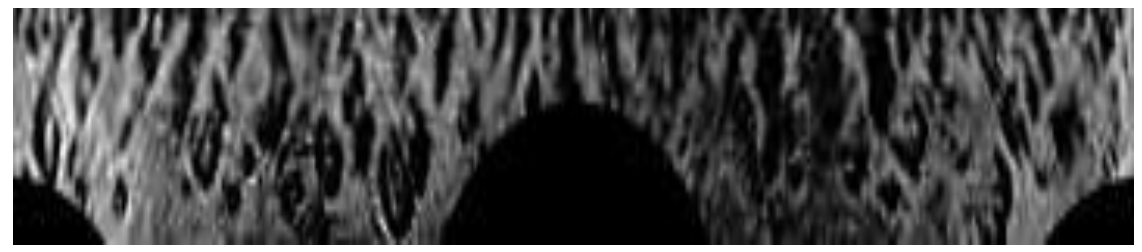

(a) 


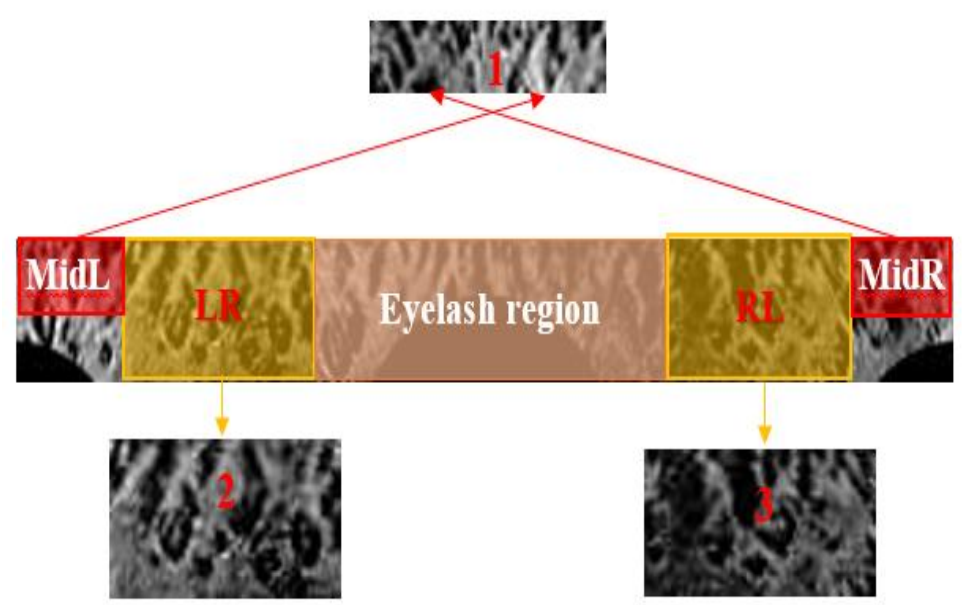

(b)

Fig 3: Normalization Operations: (a) Transform to Polar, (b) Iris Blocks

\section{FEATURE EXTRACTION OPERATION}

Important characteristics (features) will be extract from each block, so as to reduce the non-useful information in the image. Thus, templates of the matching operation contain only iris image basis that distinguish [10]. In this paper two steps are used to extract features from each block: first; Gray Level Cooccurrence Matrix (GLCM) is utilized which represent (FOS). GLCM can be defined as "a square matrix can detect a particular properties about the spatial distribution of the graylevels in the texture image"[11]. That mean each pixel value is considered reference pixel for a neighbor pixel value with a displacement vector; default $=1$, different offsets and angles has been determined the relationship between two neighboring pixels, the default has been represented between a pixel and its immediate neighbor to its right. There is four of the possible spatial relationships $(0 ; 45 ; 90$ and 135) and these offsets are: $\left\{\left[\begin{array}{ll}0 & 1\end{array}\right],\left[\begin{array}{ll}-1 & 1\end{array}\right],\left[\begin{array}{ll}-1 & 0\end{array}\right],\left[\begin{array}{ll}-1 & -1\end{array}\right]\right\}$, the details as illustrated in Figure (4).

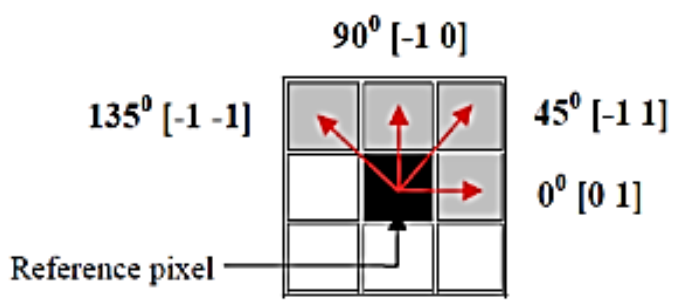

Fig 4: Co-Occurrence Matrix Directions for Extracting Texture Features

This paper executes GLCM which computed for each iris block using different values of distance, at different angle $\theta$ of offsets. The present work has experimented three types of GLCM created from using different theta, these GLCM as:

First method use two offsets with two theta as :
First distance $=$ offset $\left[\begin{array}{ll}0 & 1\end{array}\right]$
for $\theta=0$ and
Second distance $=$ offset $\left[\begin{array}{ll}-1 & 0\end{array}\right]$
for $\theta=90$

So each block gets on two matrix with size $8 * 8$ pixels, because a grayscale image is used that have 28 gray level. Thus, there are 6 matrix that enter to the next step .

Second method use three offsets with three theta as :

$$
\begin{array}{ll}
\text { First distance }=\text { offset }\left[\begin{array}{ll}
0 & 1
\end{array}\right] & \text { for } \theta=0 ; \\
\text { Second distance }=\text { offset }\left[\begin{array}{ll}
-1 & 1
\end{array}\right] & \text { for } \theta=45 \text { and } \\
\text { Third distance }=\text { offset }\left[\begin{array}{ll}
-1 & 0
\end{array}\right] & \text { for } \theta=90
\end{array}
$$

So each block gets on three matrix with size $8 * 8$ pixels. Thus, there are 9 matrix that enter to the next step.

Third method use four offsets with four theta as :

$$
\begin{array}{ll}
\text { First distance }=\text { offset }\left[\begin{array}{ll}
0 & 1
\end{array}\right] & \text { for } \theta=0 ; \\
\text { Second distance }=\text { offset }\left[\begin{array}{ll}
-1 & 1
\end{array}\right] & \text { for } \theta=45 ; \\
\text { Third distance }=\text { offset }\left[\begin{array}{ll}
-1 & 0
\end{array}\right] & \text { for } \theta=90 \text { and } \\
\text { Fourth distance }=\text { offset }\left[\begin{array}{ll}
-1 & -1
\end{array}\right] & \text { for } \theta=135
\end{array}
$$

So each block gets on four matrix with size $8 * 8$ pixels. Thus, there are 12 matrix that enter to the next step.

Secondly, set of statistical measurements from GLCM will be calculate which represented (SOS). While there are three block, the two previous steps will do for each block separately, therefore there are three vector of features lastly together in one vector.

Set (10) of statistical measurements will be calculated in the present work, these set have been derived from each GLCM for consisting a template vector that will be stored to use after that in the matching process. The set of statistical measurements that is used in this work are: Contrast, Dissimilarity, Energy, Entropy, Homogeneity, Maximum Probability, Variance, Sum Average, Inverse Difference Normalized (INN), and AutoCorrelation. These equations as $[12,13,14,15]$ :

$$
\text { Contr }=\sum_{i, j=1}^{N g}(i-j)^{2} \mathrm{C}(\mathrm{i}, \mathrm{j})
$$


Where $\mathrm{Ng}$ denotes the dimension of co-occurrence matrix (number of gray levels), and Cij represent the probability of co-occurrence between grey levels $\mathrm{i}$ and $\mathrm{j}$, which formula is defined as:

Where:

$$
C(\mathrm{i}, \mathrm{j})=\frac{\mathrm{p}_{\mathrm{ij}}}{\sum_{\mathrm{i} . \mathrm{j}=1}^{\mathrm{G}} \mathrm{p}_{\mathrm{ij}}}
$$

- pij represents the number of occurrences of gray level $\mathrm{i}$ and $\mathrm{j}$ within the given window, given a certain $(\delta, \theta)$ pair;

- $G$ is the quantized number of gray levels.

$$
\begin{gathered}
\text { Dissi }=\sum_{i, j=1}^{N_{g}}|i-j| C(i, j) \\
\text { Energ }=\sum_{i, j=1}^{N g} C(i, j)^{2}
\end{gathered}
$$

$$
\text { Homg }=\sum_{i, j=1}^{N g} \frac{1}{1+(i-j)^{2}} C(i, j)
$$$$
\operatorname{Maxpr}=\underset{i, j}{\operatorname{Max}}(C(i, j))
$$

$\operatorname{Var}=\sum_{i, j=1}^{N}|i-\mu|^{2} \mathrm{C}(i, j)$

Where $\mu$ is the mean of $\mu_{\mathrm{x}}$ and $\mu_{\mathrm{y}}$ :

$$
\begin{aligned}
& \mu_{\mathrm{x}}=\sum_{\mathrm{i}} \mathrm{i} \sum_{\mathrm{j}} \mathrm{C}(\mathrm{i}, \mathrm{j}), \mu_{\mathrm{y}}=\sum_{\mathrm{j}} \mathrm{j} \sum_{\mathrm{i}} \mathrm{C}(\mathrm{i}, \mathrm{j}) \\
& \text { SumAv }=\sum_{\mathrm{k}=2}^{2 N_{g}} \mathrm{k}_{\mathrm{x}+\mathrm{y}}(\mathrm{k}) \ldots \ldots \ldots(8) \\
& \text { Indnc }=\sum_{\mathrm{i}, \mathrm{j}=1}^{G} \frac{\mathrm{C}(\mathrm{i}, \mathrm{j})}{\left(1+|\mathrm{i}-\mathrm{j}|^{2} / \mathrm{G}^{2}\right.} \ldots \ldots \ldots(9) \\
& \text { Autoc }=\sum_{\mathrm{i}, \mathrm{j}=1}^{N g} \mathrm{ij} \mathrm{C}(\mathrm{i}, \mathrm{j}) \quad \ldots \ldots \ldots(10)
\end{aligned}
$$

\section{CREATE ARTIFICIAL NEURAL NETWORK}

After extracting the appropriate features and storing it, the recognition process will be completed, therefore an appropriate neural network must be chosen to carry out the classification models. While the network has been selected on the basis of efficiency working, thus the network must not take a long time in the training process, and avoid the networks that their performance very quickly become less efficient when increasing the number of samples. Therefore,
Probabilistic Neural Network Model (PNN) is chosen. PNN implemented as a neural network version [16] based on a Bayes-Parzen Classifier and operates by using spherical Gaussian Radial Basis Functions centered at each training vector. The PNN is an Unsupervised model, FeedForward utilized in classification and mapping of data. Generally, the previous results indicates that the PNN is more efficient than back propagation networks with respect to: classification accuracy and training time, and there is another characteristic with PNN; it is easier to add new pattern to network, so as to decrease the complexity in recognition process.

PNN composed of four layers [17]: Input Layer, Pattern Layer, Summation Layer and Output Layer. Each layer contains some neural nodes, which are the basic processing units. Where: Input Layer is an acceptor, which accepts the test vector which represent Test Pattern input to the PNN. The number of input node depend on the size of Pattern Vector, therefore three sets of features are extracted from three type of Feature Extraction, the input layer consists of:

\section{60 input node in first method of feature extraction.}

2. 90 input node in second method of feature extraction.

3.120 input node in third method of feature extraction.

Pattern Layer contains pattern neuron nodes, there is one pattern neuron node for each trained sample while each class composed of 7 samples for training. In the proposed system, three sets of image would been tested to examine the strength of the network in the recognition process, the first set contains 20 image, second set contains 25 image while the last set contains 30 image, for each set there is one model of PNN that contains the same number of pattern node, thus there are 3 models of PNN, each model consists of 3 types of PNN that are trained by using three features sets extracted in three

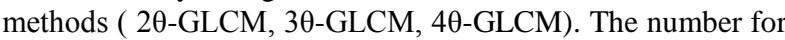
inputs nodes for each model depends on the vector type of feature method, while the number of patterns nodes depends on the number of samples for all class training in network, thus:

1. First model for 20 class we need 140 pattern node.

2. Second model for 25 class we need 175 pattern node.

3. Third model for 30 class we need 210 pattern node.

The weights between the pattern layer nodes and input layer nodes are equivalent to the probability distribution function (PDF). PNN operates by using spherical Gaussian radial basis functions centered at each training vector as PDF function. The likelihood of an unknown vector belongs to a given class can be expressed as [17]:

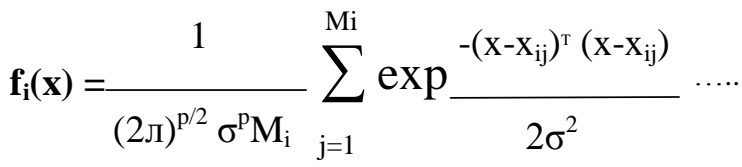

Where $\mathrm{i}$ is the class number, $\mathrm{j}$ is the pattern number, $x_{i j}$ is the $j^{\text {th }}$ training vector from class $i, x$ is the test vector, $M_{i}$ is the number of training vectors in class $i, p$ is the dimension of vector $\mathrm{x}, \sigma$ is the smoothing factor (the standard deviation), and $f i(x)$ is the sum of multivariate spherical Gaussians centered at each of the training vectors $x_{i j}$ for the $i^{\text {th }}$ class (pdf) estimate. Classification decisions are consequently made in 
accordance with the Bayes' strategy for decision rule, which is: $\mathbf{d}(\mathbf{x})=\mathbf{C i}$,

\section{if : $f i(x)>f k(x) \quad$ for $k \neq i, \quad$ Where $C i$ is the class $i$.}

Summation Layer consists of number of nodes as the same number of classes in the system, each class has one neuron in summation layer. The function of each node is to find the summation of Gaussian function for pattern neurons of each class then find the probability density function (PDF) of each class among other classes in network.PDF calculate by using this formula:

\section{$P($ class $)=\operatorname{Sum}($ neurons of class $) /$ Sum $($ classes in network $)$}

Lastly, the largest probability will be found in Output (Decision) Layer, this layer determines to which class classifies the pattern. Figure (5) displays structures of third model (30class) of PNN with three methods of feature extraction.

The network simulation (training) will be done on a set of samples for the same individuals registered in the system. After that, the network will been stored to be used in the matching (recognition) process.

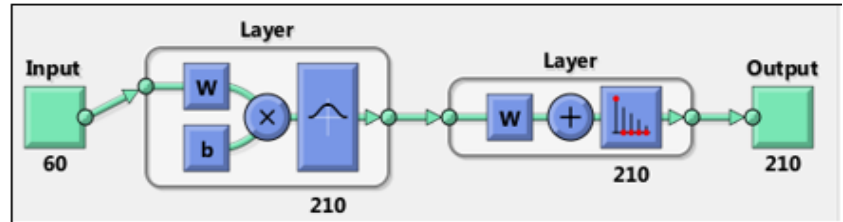

(a) Structure of PNN for First Method (2 GLCM)

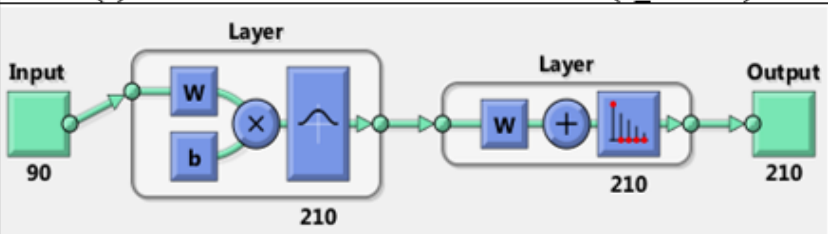

(b) Structure of PNN for Second Method (3_GLCM)

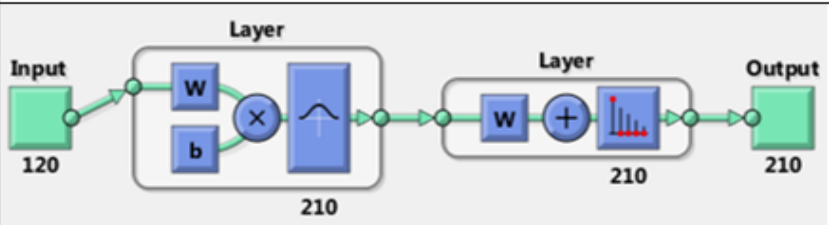

(c) Structure of PNN for Third Method (4_GLCM)

Fig 5: Structures of Third Model of PNN

\section{MATCHING OPERATION}

Matching stage requires a relating matching metric, where returns a measure of similarity degree between two iris templates one the required iris model and the other stored in the DB. Then, the decision can be made with high certainty, whether the two templates belonging to the same iris or to various irises [18]. PNN would been used in the proposed system to recognize the test pattern. In recognition stage, the system will receive an image for individual who will recognize it, then extracted features vector from this image by using all operations of preprocessing and feature extraction. Thus, the Test Pattern enter for the training PNN to determine to which class it belongs and make a decision to (accept/ reject) an individual. In the proposed system, at least Three Samples are used for each class as Test Patterns in recognition process, Notice Test Samples different from the Trained Samples for the same class. The output of matching algorithm would have been the name of class which entering sample belong it, if it had previously registered in the registration process.

\section{EXPERIMENTAL RESULT}

The subject significance of this paper is to increase the accuracy of the IRS by using Statistical Measurements calculated from GLCM with PNN. The efficiency of the system will be evaluated by utilizing iris images of CASIA dataset. The system application will be under environment of "windows 7 Ultimate" with processor Intel "Core ${ }^{(\mathrm{TM})} \mathrm{i} 5$ ". The proposed system consists of three stages: the first stage is a registration process, in this stage images taken from dataset are used, each individual in this dataset at least have 10 samples. So, for each class 7 samples used in training the network and the rest samples of same individual used for testing in matching process. Each registration and matching process preprocessing operations are conducted and give high accuracy, the result of it be 3 blocks as: First (Down) Block with size $18^{*} 84$, while Second (Left) and Third (Right) Blocks with size $35^{*} 70$, then extract features from each block separately. Feature extraction process experiments three methods: The First Method two angles are taken of offsets $(0$, 90) to obtain two GLCM, in Second Method three angles are taken of offsets $(0,45,90)$ to obtain three GLCM, while in Third Method four angles are taken of offsets $(0,45,90,135)$ to obtain four GLCM. For each GLCM (10) statistical measurements would been calculated to get on features vector. The last step in registration process will store features extracted in database to be used in the next stage (Training Stage). The second stage builds three models of recognition system depending on the number of classes in PNN $(20,25,30)$, for each model a group of samples is training for all classes ( 7 patterns for each class) by using three types of features extracting which depends on the number of GLCM (according to number of offsets angles). Table (1) describes the number of inputs for training each model of PNN with each method of feature extraction.

Tab 1: Inputs for Each Model of PNN

\begin{tabular}{|c|c|c|c|}
\hline Method & & o. of Training in & puts \\
\hline Model & $\begin{array}{l}\text { First Method } \\
(2 G L C M)(60)\end{array}$ & $\begin{array}{l}\text { Second Method } \\
(3 G L C M)(90)\end{array}$ & $\begin{array}{l}\text { Third Method } \\
(\text { (4GLCM)(120) }\end{array}$ \\
\hline $\begin{array}{l}\text { First Model } \\
(20 * 7)=140\end{array}$ & 8,400 & 12,600 & 16,800 \\
\hline $\begin{array}{l}\text { Second Model } \\
(25 * 7)=175\end{array}$ & 10,500 & 15,750 & 21,000 \\
\hline $\begin{array}{l}\text { Third Model } \\
(30 * 7)=210\end{array}$ & 12,600 & 18,900 & 25,200 \\
\hline
\end{tabular}

PNN is trained by using Gaussian function as activation function of network with select an appropriate value of smoothing factor $\sigma$ to be suitable for all samples. Gaussian function depends on the smoothing factor (standard deviation $\sigma)$ that controls the smoothing degree of image, this factor must be accurately chosen to ensure not losing the details in features and at the same time find good approximation for each feature (don't spiky). Therefore an appropriate factor should be found for smoothing image to protect the distinct features for each pattern which makes a pattern for all samples distinct from pattern of other samples. So, the benefit from the First (20 class) and Second ( 25 class) Models is to find the best value of the smoothing factor. In the First Model; 9 values for $\sigma$ were tested as: $[0.1 \ldots 0.9]$, while in the Second Model (25 class); 5 values for $\sigma$ were tested are: $[0.1 \ldots 0.6]$. Therefore, Third Model (30 class) is tested with best three values of $\sigma[0.3,0.4,0.5]$ (values given high rates of recognition). 
Two Measures are adopted to indicate the accuracy of the results: the first one is True Acceptance Rate (TAR) or Recognition Rate, and False Rejection Rate (FRR).

TAR $=\frac{\text { No. of True Acceptance }}{\text { Total Number of Samples }} * 100$

FRR $=$ No. of False Rejection $* 100$

The results of testing First Models of PNN which is displayed in table (2) that in 2GLCM and 3GLCM Methods of Features Extraction, a good (TAR) is resulted from using 0.4 value of $\sigma$, while the best rate (TAR) from using 4GLCM method obtained from using $\sigma$ value $=0.5$. While (FRR) gradually decreases in this range of $\sigma$ values for all three methods. The (TAR) obtained from using 4GLCM method represent the best result from the whole results.

Tab 2: TAR and FRR for First Model using Five Value of $\sigma$ and Three Types of Features Extraction

\begin{tabular}{|c|c|c|c|c|c|c|}
\hline \multirow{2}{*}{$\begin{array}{c}\text { No. of } \\
\text { S.F.(6) }\end{array}$} & \multicolumn{2}{|c|}{$\begin{array}{c}\text { First Method } \\
\text { (2 GLCM) }\end{array}$} & \multicolumn{2}{c|}{$\begin{array}{c}\text { Second Method } \\
\text { (3 GLCM) }\end{array}$} & \multicolumn{2}{c|}{$\begin{array}{c}\text { Third Method } \\
\text { (4 GLCM) }\end{array}$} \\
\cline { 2 - 7 } & TAR & FRR & TAR & FRR & TAR & FRR \\
\hline $\mathbf{0 . 1}$ & $88.76 \%$ & $11.24 \%$ & $82.73 \%$ & $17.27 \%$ & $77.51 \%$ & $22.49 \%$ \\
\hline $\mathbf{0 . 2}$ & $96.39 \%$ & $3.61 \%$ & $95.58 \%$ & $4.42 \%$ & $95.18 \%$ & $4.82 \%$ \\
\hline $\mathbf{0 . 3}$ & $97.99 \%$ & $2.01 \%$ & $97.99 \%$ & $2.01 \%$ & $97.19 \%$ & $2.81 \%$ \\
\hline $\mathbf{0 . 4}$ & $98.39 \%$ & $1.61 \%$ & $98.80 \%$ & $1.20 \%$ & $99,20 \%$ & $0.80 \%$ \\
\hline $\mathbf{0 . 5}$ & $98.39 \%$ & $1.61 \%$ & $98.80 \%$ & $1.20 \%$ & $100 \%$ & $0.00 \%$ \\
\hline
\end{tabular}

The results of testing The Second Models of PNN Which is displayed in table (3), so the analysis of the rates would prove that $\sigma$ value 0.3 is an appropriate smoothing factor for PNN with first method (2GLCM) of features extraction. As well as, the second method (3GLCM) obtained the best result at $\sigma$ value 0.4 and stable. While in third method (4GLCM) best rate (TAR) at $\sigma$ value 0.5 , while (FRR) gradually decreases in this range of $\sigma$ values for the whole three methods. The higher Rate of Recognition (TAR) would been obtained from using the third method of feature extraction.
Tab 3: TAR and FRR for Second Model using Five Values of $\sigma$ and Three Types of Features Extraction

\begin{tabular}{|c|c|c|c|c|c|c|}
\hline \multirow{2}{*}{$\begin{array}{c}\text { No. of } \\
\text { S.F.(6) }\end{array}$} & \multicolumn{2}{|c|}{$\begin{array}{c}\text { First Method } \\
\text { (2 GLCM) }\end{array}$} & \multicolumn{2}{c|}{$\begin{array}{c}\text { Second Method } \\
\text { (3 GLCM) }\end{array}$} & \multicolumn{2}{c|}{$\begin{array}{c}\text { Third Method } \\
\text { (4 GLCM) }\end{array}$} \\
\cline { 2 - 7 } & TAR & FRR & TAR & FRR & TAR & FRR \\
\hline $\mathbf{0 . 1}$ & $86.38 \%$ & $13.62 \%$ & $82.39 \%$ & $17.61 \%$ & $77.41 \%$ & $20.93 \%$ \\
\hline $\mathbf{0 . 2}$ & $92.69 \%$ & $7.31 \%$ & $93.02 \%$ & $6.98 \%$ & $93.02 \%$ & $6.98 \%$ \\
\hline $\mathbf{0 . 3}$ & $94.02 \%$ & $5.98 \%$ & $94.35 \%$ & $5.65 \%$ & $94.68 \%$ & $5.32 \%$ \\
\hline $\mathbf{0 . 4}$ & $94.02 \%$ & $5.98 \%$ & $94.68 \%$ & $5.32 \%$ & $95.68 \%$ & $4.32 \%$ \\
\hline $\mathbf{0 . 5}$ & $94.02 \%$ & $5.98 \%$ & $94.68 \%$ & $5.32 \%$ & $96.01 \%$ & $3.99 \%$ \\
\hline
\end{tabular}

Noted from Charts in Figure (6) which displays TAR, FRR for First and Second Model of PNN. That, number of classes increases and the type of features extraction influenced by smoothing factor $\sigma$, while the best result obtained from using 4 GLCM method. To prove this fact, these three methods of features extraction (2ang.GLCM, 3ang.GLCM and 4ang.GLCM) are tested using third Model of PNN which contains a large number of classes. While two previous models are found to have three values of smoothing factor $\sigma$ $[0.3,0.4,0.5]$ given the best rate, therefore Third Model with these three $\sigma$ values is tested, as shown in Fig.(6) which displays charts of applying range values of $\sigma[0.1 \ldots 0.5]$ on TAR and FRR in First and Second Model .

The results of Recognition Rate (TAR) and (FRR) of Third Model of PNN with 30 classes and three method of feature extraction (2GLCM, 3GLCM and 4GLCM) with three values of smoothing factor $\sigma[0.3,0.4$ and 0.5]. So, in the First Method (2_GLCM) the best Gaussian value of training PNN would be 0.3, while in the Second Method (3_GLCM) would be 0.4 , and in the Third Method of Feature Extraction (4_GLCM) would be 0.5 , while it is noticed that False Rejection Rate (FRR) would decrease with the inverse format of (TAR) as shown in Chart in Figure (7).

Tab 4: TAR and FRR for Third Model using Three Values of $\sigma(0.3,0.4$, and 0.5$)$ and Three Types of Features Extraction

\begin{tabular}{|c|c|c|c|c|c|c|}
\hline \multirow{2}{*}{$\begin{array}{l}\text { No. of } \\
\text { S.F.( }(\sigma)\end{array}$} & \multicolumn{2}{|c|}{$\begin{array}{c}\text { First Method } \\
(2 \text { GLCM) }\end{array}$} & \multicolumn{2}{|c|}{$\begin{array}{c}\text { Second Method } \\
\text { (3 GLCM) }\end{array}$} & \multicolumn{2}{|c|}{$\begin{array}{c}\text { Third Method } \\
\text { (4 GLCM) }\end{array}$} \\
\hline & TAR & FRR & TAR & FRR & TAR & FRR \\
\hline 0.3 & $91.09 \%$ & $8.91 \%$ & $93.04 \%$ & $6.96 \%$ & $93.31 \%$ & $6.69 \%$ \\
\hline 0.4 & $91.09 \%$ & $8.91 \%$ & $93.31 \%$ & $6.69 \%$ & $94.15 \%$ & $5.85 \%$ \\
\hline 0.5 & $91.09 \%$ & $8.91 \%$ & $93.31 \%$ & $6.69 \%$ & $94.43 \%$ & $5.57 \%$ \\
\hline
\end{tabular}




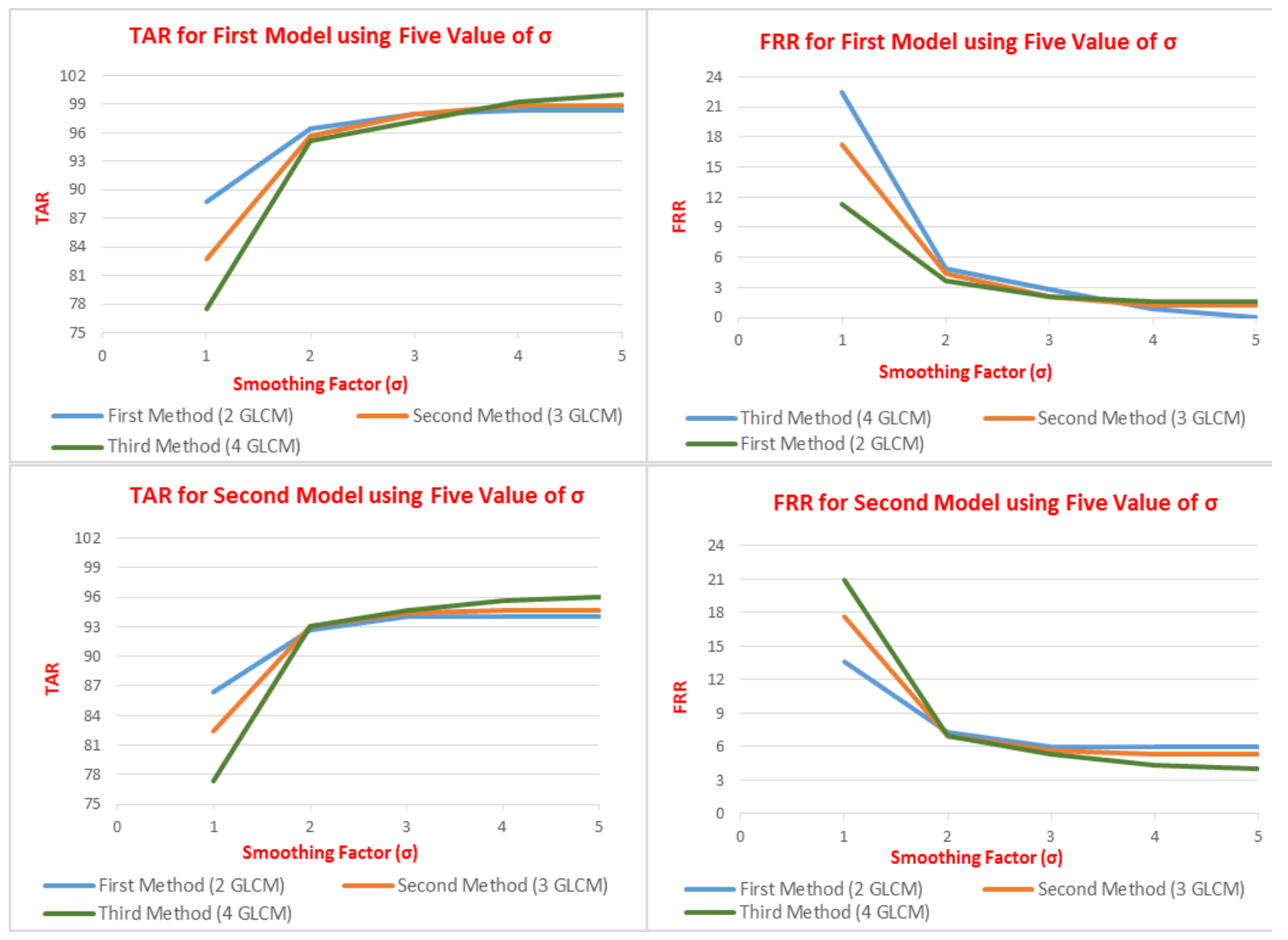

Fig 6: Effect of Applying Range Values of $\sigma[0.1 \ldots$ 0.5] on TAR and FRR in First \& Second Model
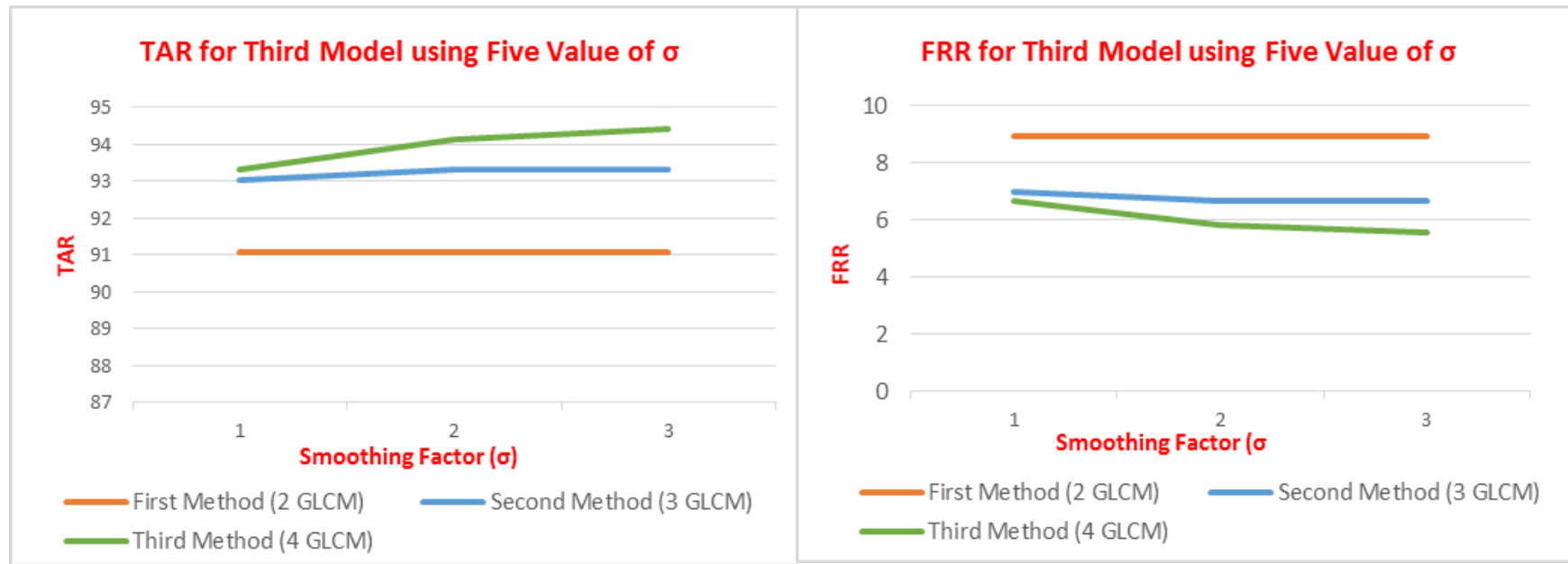

Fig 7: Effect of Applying Three Values of $\sigma(0.3,0.4,0.5)$ on TAR and FRR in Third model

\section{CONCLUSION}

This paper presents a comparison between three types of feature extraction with PNN used in matching operation an IRS. After all preprocessing operations finished, ROI isolated from iris image, a new method is used to crop three blocks from ROI, then extract features from each block separately. In feature extraction process, three types of GLCM based on a number of angles (2Ang.GLCM, 3Ang.GLCM, 4Ang.GLCM) are extracted as FOS then 10 statistical measurements are collected as SOS. Three models of PNN is used depending on a number of classes $(20,25,30)$ to prove the efficiency of PNN with the kind of feature extraction method, while an appropriate control parameter (smoothing factor $\sigma$ ) in activation function of PNN will be chosen by experimenting serial values of $\sigma$ with first (20 class) and second ( 25 class) models to prove that best Recognition Rate gets from using 
0.3 value of smoothing factor $\sigma$ with first method (2_GLCM), 0.4 value of $\sigma$ with second method ( 3 GLCM), and 0.5 value of $\sigma$ with third method (4_GLCM). That fact is proven from training PNN with third model (30 class) with three methods of feature extraction. Experimentally, the Recognition Rate of Third Model showed that best (TAR) would be obtained from using the Third Method of Feature Extraction (4GLCM) trained with 0.5 value of $\sigma$ to get a $94.43 \%$ accuracy. Thus, this method proved that it is an appropriate method with PNN to make the proposed system more efficiency.

\section{REFERENCES}

[1] R. H. Abiyev and K. Altunkaya, "Personal Iris Recognition Using Neural Network", International Journal of Security and its Applications, Vol. 2, No. 2, April 2008

[2] C. Jayachandra and H. V. Reddy, "Iris Recognition based on pupil using Canny Edge Detection and K-Mean algorithm", International Journal of Engineering and Computer science ISSN: 2319-7242, Vol. 2, No. 1, Pp. 221-225, 1 Jan 2013

[3] T. Karthikeyan, "Efficient Bio Metric IRIS Recognition System Using Fuzzy Neural Network", Int. J.of Advanced Networking and Application, Vol. 01, No. 06, Pp. 371-376, 2010

[4] A. K. Jain, A. Ross and S. Prabhakar, "An Introduction to Biometric Recognition", IEEE Transactions on Circuits and Systems for Video Technology, Special Issue on Image- and Video-Based Biometrics, Vol.14, No. 1, Jan. 2004.

[5] M. Elgamal and N. Al-Biqami, "An Efficient Feature Extraction Method for Iris Recognition based on Wavelet Transformation", International Journal of Computer and Information Technology ISSN: 2297-0764, Vol. 02, No. 03, 1May 2013.

[6] A. Murugan and G. Savithiri, "Fragmented Iris Recognition System using BPNN", International Journal of Computer Applications, Vol. 36-No.4, Dec. 2011.

[7] L. Yang, F. Albregtsen, T. Lgnnestad, and P. Grgttum, " Methods to Estimate Areas and Perimeters of Blob-Like Objects: A Comparison", IAPR Workshop on Machine Vision Applications, Pp.13-15, 1994.

[8] K. Roy, P. Bhattacharya and R. C. Debnath, "Multi-Class SVM Based Iris Recognition", Computer and information technology, iccit2007. 10th international conference on. IEEE, Pp. 1-6, 2007.

[9] M. Vatsa, R. Singh and A. Noore, "Reducing the False Rejection Rate of Iris Recognition Using Textural and Topological Features", International Journal of Signal Processing, ISSN: 1304 - 4494, Vol. 2, No.2, 2005.

[10] S. V. Sheela, and P. A. Vijaya, "Iris Recognition Methods - Survey" International Journal of Computer Applications, Vol. 3, No. 5, Pp. 19-25, June 2010.

[11] B. Pathak, and D. Barooah, "Texture Analysis Based on the Gray-Level Co-Occurrence Matrix Considering Possible Orientations", International Journal of Advanced Research in Electrical; Electronics and Instrumentation Engineering, Vol. 2, No. 9, Sep.2013.

[12] D. Gadkari, "Image Quality Analysis Using GLCM", thesis submitted to the College of Arts and Sciences / University of Central Florida, 2004

[13] D. A. Clausi,"An analysis of co-occurrence texture statistics as a function of grey level quantization", Canadian Journal of remote sensing, Vol. 28, No. 1, pp. 45-62, 2002.

[14] L. Soh, and C. Tsatsoulis,"Texture Analysis of SAR Sea Ice Imagery Using Gray Level Co-Occurrence Matrices", IEEE Transactions On Geoscience And Remote Sensing, Vol. 37, No. 2, Mar. 1999

[15] D. A. Kumar, and S. Dhandapani ,"A Bank Cheque Signature Verification System using FFBP Neural Network Architecture and Feature Extraction based on GLCM", International Journal of Emerging Trends \& Technology in Computer Science (IJETTCS), Vol. 3, No. 3, 2014 .

[16] R. Low, and R. Togneri. "Speech recognition using the probabilistic neural network." ICSLP, 1998.

[17] X. Wu, Fang Lu, Bo Wang, and Jingzhi Cheng, "Analysis of DNA sequence pattern using probabilistic neural network model", Journal of Research and Practice in Information Technology, Vol. 37, No. 4, Pp. 353-363, Nov. 2005.

[18] H. R. Sushma, and R. Sandeep, "Multi Biometric Template Protection using Random Projection and Adaptive Bloom Filter", International Journal of Research in Electronics and Computer Engineering (IJRECE), VOL. 3, No. 2, June 2015. 